Revue d'histoire de l'Amérique française

AHE REVUE D.HISTOIRE DE L'AMÉRIQUE FRANÇAISE

\title{
Le Récit de M. de Courcelles au lac Ontario (1671), et Dollier de Casson
}

Jean Marmier

Volume 32, numéro 2, septembre 1978

URI : https://id.erudit.org/iderudit/303692ar

DOI : https://doi.org/10.7202/303692ar

Aller au sommaire du numéro

Éditeur(s)

Institut d'histoire de l'Amérique française

ISSN

0035-2357 (imprimé)

1492-1383 (numérique)

Découvrir la revue

Citer cette note

Marmier, J. (1978). Le Récit de M. de Courcelles au lac Ontario (1671), et Dollier de Casson. Revue d'histoire de l'Amérique française, 32(2), 239-250.

https://doi.org/10.7202/303692ar d'utilisation que vous pouvez consulter en ligne.

https://apropos.erudit.org/fr/usagers/politique-dutilisation/ 


\section{LE RÉCIT DE M. DE COURCELLES \\ AU LAC ONTARIO (1671), ET DOLLIER DE CASSON}

JEAN MARMier

Rennes

Le Récit de ce qui s'est passé au voyage que Monsieur de Courcelles Gouverneur de la Nouvelle France a fait au Lac Ontario, ou, des Irocquois ne figure pas au nombre des narrations canadiennes du $\mathrm{XVII}^{\mathrm{e}}$ siècle les plus connues. Il ne manque pas d'intérêt pourtant. Il pose d'abord un problème d'attribution, dont nous exposerons les données après avoir brièvement présenté le document.

\section{Les faits}

On sait que le gouverneur Rémy de Courcelles, arrivé à Québec en 1665 en compagnie de Talon, et précédé de peu par Tracy, eut d'abord à se préoccuper de la menace iroquoise. Dès janvier 1666, il menait contre les Agniers une expédition qui tourna mal. En septembre 1666, il repartait avec Tracy pour réparer cet échec; on put cette fois crier victoire, sur un très maigre bilan, mais non sans motif, puisqu'une période de paix s'ensuivit. Les Iroquois n'en demeuraient pas moins la hantise de la colonie. On restait exposé à un péril perpétuel à Montréal, et, à plus forte raison, dans les territoires isolés. Du point de vue économique, non contents de vendre leurs pelleteries aux Hollandais et aux Anglais, les Iroquois attaquaient en 1670 les Algonquins et les Outaouais, habituels fournisseurs des Français. Courcelles éprouvait quelque peine à arrêter cette guerre et à imposer l'échange des prisonniers. Mais, aussitôt, les Iroquois essayaient par la négociation de détourner la traite des Outaouais 
vers la Nouvelle-Hollande: manœuvre dont le succès eut été catastrophique pour la colonie française. C'est afin de les intimider que le gouverneur entreprit un «voyage» militaire, mais pacifique. Avec une soixantaine d'hommes, il monta de Montréal jusqu'à l'extrémité orientale du lac Ontario en empruntant la voie fluviale à partir du Sault Saint-Louis. Partie le 2 juin, la petite troupe était de retour le 17 du même mois. Le fait essentiel de l'opération, c'est qu'on avait mené jusqu'au-dessus d'Otondiata, qui marquait la fin de la série des rapides du Saint-Laurent, non seulement des canots d'écorce, mais un bâtiment plat à l'européenne, de deux ou trois tonneaux, et qu'on avait ramené le tout, sans accident sinon sans difficultés, à la stupéfaction des Iroquois. Ceux-ci se fiaient, en effet, aux rapides pour barrer l'accès de leur pays à une armée française munie de lourds équipements et capable d'instaurer un contrôle sur le lac. La rapidité du trajet formait un élément de la démonstration. On obtint donc l'effet psychologique escompté et on préserva momentanément le circuit commercial. Dès 1672 Frontenac allait reprendre la même politique: il monta à son tour au lac Ontario avec deux bateaux de même type et y fonda le prétendu «fort» auquel il donna son nom.

\section{Le texte}

Le récit détaillé du voyage de 1671 fut rédigé à bref délai. Il enregistre encore l'arrivée des Outaouais avec leurs pelleteries «peu de jours après le retour», et, "ensuite», celle de plusieurs missionnaires venant des villages iroquois. Mais il se date explicitement de l'année 1671 même ${ }^{1}$. Il a été écrit à Montréal très probablement, d'après cette mention: «Il y a deux ans qu'il partit d'ici deux ecclésiastiques...»: c'est de Montréal qu'étaient partis, le 6 juillet 1669, les sulpiciens Dollier de Casson et Bréhant de Galinée, d'abord accompagnés par Cavelier de La Salle, pour se rendre aux Grands Lacs $^{2}$.

1 «Dès l'automne de l'an dernier $1670 \ldots », 190$. Nous citons le Récit d'après l'édition Margry, Découvertes et Établissements... I, malgré les réserves indiquées plus loin.

2 Voir le récit de ce voyage par Bréhant de Galinée (éd. Margry) ibid.: 112166 ou de préférence dans l'édition-traduction de James H. Coyne, Exploration of the Great Lakes by Dollier de Casson and de Bréhant de Galinée. Galinée's narrative and map with an English version, including all the map legends, parue dans les Ontario Historical Society Papers and Records, IV (1903). Verreau s'était procuré une édition pour la Société historique de Montréal en 1875. 
Selon l'auteur, le récit est destiné à un usage interne. Il doit circuler en Nouvelle France: «...il a été important de mettre devant les yeux des peuples de cette colonie les soins et les fatigues que $\mathbf{M}$. le Gouverneur prend pour leur conservation, afin de les rendre plus affectionnez à lui tesmoigner des obéissances qu'ils lui doivent. ${ }^{3}$ En réalité, il nourrissait une ambition différente, car à quoi bon donner aux habitants de la colonie des explications élémentaires sur les difficultés de la navigation dans les rapides et leur rappeler que les Européens se trouvent engagés dans des luttes contre «un peuple de sauvages appelé les Iroquois»? De toute évidence, on envisageait de faire parvenir le récit en France, où il a fini par arriver, à une date inconnue, peut-être dès l'automne 1671. C'est là surtout qu'il aurait pu servir utilement la réputation de Courcelles. Tel était bien son but, comme le prouvent maints passages qui rehaussent le rôle personnel du gouverneur ${ }^{4}$.

Le texte n'a été publié qu'une seule fois en français, en 1879 , par Pierre Margry, au tome I de ses Découvertes et Établissements des Français dans l'Ouest et le Sud de l'Amérique septentrionale, 1614-1698. Auparavant une traduction anglaise avait paru dans les Documents relating to the colonial history of the State of New York (Albany, 1855; IX: 75-94). Le manuscrit primitif est conservé à la Bibliothèque Nationale de Paris ( $\mathrm{n}^{\circ}$ 13.I56 de l'Ancien Fonds français, fos 207-218) ${ }^{5}$. À la même Bibliothèque existe une copie manuscrite ( ${ }^{\circ} 9.288$ des Nouvelles Acquisitions françaises, p. 79-87), dressée au XIX ${ }^{\mathrm{e}}$ siècle pour Margry. Il ne fait pas de doute que le manuscrit 13.156 ne constitue l'original, en raison des nombreuses corrections portées au fil du texte ou en marge. Toutes ces corrections sont de la même main que le corps du texte. L'édition de Margry reproduit assez fidèlement le manuscrit. Cependant, comme il arrive souvent, l'archiviste de la Marine a pris quelques libertés qui justifieraient une nouvelle édition plus scrupuleuse ${ }^{6}$.

3 Ibid., 169.

4 Par exemple, p. $178,182,188 \ldots$

5 Le volume contient de nombreux textes, de longueur et d'époques diverses, sur le Canada. Le premier feuillet porte une dédicace à l'abbé de Pelletier par Belmont; mais elle se rapporte au premier texte, intitulé Histoire du Canada, et non à l'ensemble de ce recueil factice. L'écriture du Récit qui nous concerne ne ressemble à aucune autre dans le recueil.

6 Parfois l'éditeur subsitue un synonyme par inadvertance («avec mauvaise foy», p. 179, pour «avec fraude», ms. $\mathrm{f}^{\circ} 211 \mathrm{v}^{\circ}$ ); parfois l'auteur a biffé un passage pour le récrire en marge sous une forme différente; mais la seconde version, rognée à la reliure, étant peu déchiffrable, l'éditeur reproduit la première, restée lisible, sans avertir le lecteur (p. 174, ms. f $^{\circ} 209 \mathrm{r}^{\circ}$ ). 
Le manuscrit est anonyme. Ni le traducteur américain de 1855 ni l'éditeur français n'ont proposé d'attribution. La question n'est pourtant plus entièrement vierge. Certains l'ont supposée résolue. Mais elle n'a jamais fait l'objet d'une discussion. Or elle baigne dans une obscurité qui prête à réflexion.

\section{L'auteur: l'hypothèse Dollier}

Qui cherche à l'éclaircir se tourne spontanément vers l'hypothèse la plus défendable. François Dollier de Casson, prêtre de Saint-Sulpice, participait au voyage en qualité d'aumônier. C'est au retour qu'il allait devenir supérieur du Séminaire de Montréal, troisième du titre. Le Récit mentionne son nom, ajouté en marge par le scripteur ${ }^{7}$. Qu'il en soit l'auteur paraît une idée si plausible que plusieurs érudits notoires ont admis d'office sa paternité.

Le premier d'entre eux fut l'abbé Étienne-Michel Faillon, dans son Histoire de la colonie française au Canada ${ }^{8}$. Sans se soucier plus que lui de la justifier, les rares historiens qui ont fait référence au Récit en indiquant un nom d'auteur ont suivi l'abbé Faillon: au $\mathrm{XIX}^{\mathrm{e}}$ siècle, le sulpicien Gamon dans une notice manuscrite ${ }^{9}$ et, de nos jours Lionel Groulx ${ }^{10}$. Si Henri Omont l'a inscrit également à l'actif de Dollier en répertoriant les manuscrits de la Bibliothèque Nationale ${ }^{11}$ et s'il a entraîné à sa suite l'auteur de la Bibliothèque sulpicienne ${ }^{12}$, c'est cependant pour une autre raison. En effet, la copie dressée pour Margry (n.a.9.288) comporte des annotations. En marge, on se demande qui est «celui qui écrit. Serait-ce La Salle? Jolliet? Qui ? ou Belmont?», et une autre main ajoute: «C'est Dollier ${ }^{13}$. Sur la page-titre, une note ultérieure confirme: "Le mémoire doit être de M. Dollier, de Saint-Sulpice, qui fit avec La Salle et M.

7 Ibid., 183, ms. f $213 \mathrm{r}^{\circ}$.

8 (1865), III : 330-336 (François-Xavier Garneau signale le voyage, mais non le Récit : Histoire du Canada ... (4 $4^{\circ}$ éd., 1882), I: 217.

9 Notice sur Dollier de Casson, inédite, conservée au Séminaire de SaintSulpice à Paris. Nous remercions M. Irénée Noye, archiviste de Saint-Sulpice, de l'obligeance avec laquelle il a facilité nos recherches.

10 Notre grande aventure. L'empire français en Amérique du Nord (15351760) (1958), 122, 131.

11 Catalogue général des manuscrits... Ancien supplément français, III : 70.

12 Louis Bertrand, Bibliothèque sulpicienne ou Histoire littéraire de la Compagnie de Saint-Sulpice (1900), I: 160.

$13 \quad$ N.a.9.288, $\mathrm{f}^{\circ} 80 \mathrm{v}^{\circ}$. 
Galinée les premières découvertes vers le Sud de l'Amérique du Nord ${ }^{14}$. D'autre part, le manuscrit original (Anc.supp.fr.13.156) est précédé de l'indication suivante: "Il paraît que le Récit qui suit est de la main de M. l'abbé Dollier, compagnon de Cavelier de La Salle en 1669-70, d'après son rapprochement du fac-similé d'une lettre autographe. 1844.»

L'origine de ces annotations d'écritures diverses remonte évidemment à Margry et, sans doute, à son ami l'abbé Faillon. Nous reviendrons sur la comparaison avec les autographes de Dollier. Ajoutons encore que Ralph Flenley, éditeur et traducteur de l'Histoire du Montréal de Dollier, recourt, lui, à l' «évidence interne» pour attribuer «presque certainement» le Récit à Dollier, mais ne donne pas plus de détails ${ }^{15}$.

Toutefois, en face de ces autorités, s'alignent les principaux biographes du sulpicien. S'ils connaissent sa participation au voyage de 1671, ils ignorent qu'il en ait écrit le récit. Tel était déjà le cas de son contemporain et ami Joseph Grandet, supérieur du Séminaire d'Angers, puis celui de Lobineau. Tel reste le cas d'érudits comme Jean Domberval (Henri Gauthier), de chercheurs aussi informés que le chanoine Uzureau, Olivier Maurault, Jacques Mathieu, W.J. Eccles, et d'amateurs comme Robert de Roquebrune ${ }^{16}$. Faut-il leur imputer une omission, ou les louer de leur prudence? Certaines observations peuvent nourrir la discussion et voici d'abord celles qui plaideraient en faveur de l'attribution du Récit à Dollier.

\section{Discussion}

À peine débarqué au Canada, Dollier, frais émoulu de SaintSulpice mais ancien officier de cavalerie, avait déjà accompagné

$14 \mathrm{~F}^{\circ} 79 \mathrm{r}^{\circ}$.

15 A History of Montreal. 1640-1672, translated and edited with a life of the author (Toronto, 1928) 14.

16 Lobineau, Les Vies des Saints de Bretagne et des personnes d'une éminente piété qui ont vécu dans cette province (1838), V: 305 sqq., reproduit la notice manuscrite de Joseph Grandet. - Jean Domberval, Archives et souvenirs (1938), 31. - F. Uzureau, «L'abbé Dollier de Casson, Supérieur du Séminaire de Montréal», dans Le Canada français (1925): 134-139. - Oliver Maurault, «Études sur Dollier de Casson" dans la Revue trimestrielle canadienne, IV (1919): 361-370. — Jacques Mathieu, "Dollier de Casson», dans le Dictionnaire, biographique du Canada, II - W. J. Eccles, «Rémy de Courcelle (s)», ibid., - Robert de Roquebrune, "Deux historiens de Montréal au XVIIe siècle», dans Le Canada français (oct. 1933) : 19-30. 
Courcelles et Tracy dans la campagne de l'automne 1666 contre les Agniers. Si, en 1671, Courcelles s'était décidé à monter au lac Ontario, c'était, nous explique l'auteur, entre autres raisons, parce que le voyage accompli deux ans auparavant par «deux ecclésiastiques» avait attiré l'attention sur un passage éventuel en direction de l'Ouest par le cours de la "grande rivière», c'est-à-dire l'OhioMississipi, que l'on supposait couler vers la Nouvelle-Espagne. Or, pour gagner la grande rivière, l'itinéraire le plus commode passait par le lac Ontario. Une longue expédition missionnaire avait, en effet, conduit, en 1669-70, Dollier et son ami Galinée au lac Ontario, puis au lac Érié, à l'ouest duquel ils avaient hiverné, au lac Huron, et jusqu'à Sainte-Marie-du-Sault. Une phrase importante du Récit de 1671 assure que "celuy qui escrit ne rapporte que ce qu'il a veu» ${ }^{17}$. Cette phrase concerne la description des Grands Lacs qui introduit la narration et ne concerne peut-être qu'elle. Bien que Dollier n'eût pas dépassé le Sault Sainte-Marie, il était fondé à invoquer son expérience personnelle pour décrire le circuit des lacs.

Il possédait bien entendu les capacités requises pour rédiger un tel récit et ne répugnait pas à les utiliser. Nous savons qu'il avait composé un compte rendu de son grand périple, mais qu'il l'avait éliminé au profit de son ami Galinée, qui lui semblait de meilleur style ${ }^{18}$. Il allait écrire en 1672 son Histoire du Montréal, en vue de laquelle il avait vraisemblablement déjà rassemblé des notes. Il la laisserait d'ailleurs anonyme et inédite, comme le Récit. Il allait y résumer la démonstration de 1671 au lac Ontario. Il y insisterait, comme l'auteur du Récit, sur le secret soigneusement gardé par Courcelles et levé seulement après l'arrivée du gouverneur à Montréal, ainsi que sur les heureux effets de l'opération ${ }^{19}$. Dans ces convergences réside peut-être l' «évidence interne» dont parle Flenley.

Dollier entretenait d'excellentes relations avec Courcelles qui, en quittant le Canada l'année suivante, lui laissa la tutelle d'une petite fille iroquoise qu'il avait adoptée, avec une somme de mille livres. La méfiance des autorités civiles envers les Jésuites - Courcelles aussi bien que Talon - renforçait d'ailleurs le crédit des Sulpiciens auprès d'elles. On imagine aisément que le gouverneur ait prié

\footnotetext{
(Récit): 171.

Histoire du Montréal (éd. Flenley), 336.

Ibid., 340-342.
} 
le nouveau supérieur ou lui ait suggéré de raconter l'événement d'importance notable auquel il venait de participer, ou que Dollier l'ait entrepris spontanément. La manière neutre selon laquelle sa présence est signalée ( Un Ecclésiastique du Montréal, nommé $\mathbf{M}$. Dolier...») rappelle celle dont l'auteur use quant il s'introduit, rarement cependant, dans l'Histoire de Montréal ${ }^{20}$.

Parallèlement aux analogies que présente cependant le texte avec celui de l'Histoire du Montréal, on en constate d'autres avec le texte de la relation de Galinée. Elle était inédite, mais Dollier l'avait évidemment lue de près et préférée à son propre récit. Que ces analogies portent sur la description des canots d'écorce des sauvages, fort admirés par Galinée ${ }^{21}$, et sur le supplice réservé par eux aux prisonniers, leur ôteraient toute signification, puisqu'il s'agit là de «topoï» du genre, si le démarcage ne semblait littéral. Citons le Récit sur les canots «si volages que dix livres d'un côté plus que de l'autre les font tourner, ce qui oblige à s'y tenir à genoux ou assis tout à plat», et Galinée: "Il faut se tenir tout le temps qu'on est dans ces canots à genoux ou assis, prenant garde de bien garder l'équilibre, car ces bastimens sont si légers, qu'un poids de vingt livres sur un bord plus que sur l'autre est capable de les faire tourner.» Dans les deux cas, leur grande fragilité est associée à cette remarque. Dans le Récit, on brûle les prisonniers, "attachez pieds et mains à un pilier », avec un «vieux canon de fusil tout rouge», "en sorte qu'il ne reste aucun endroit de leur corps qui ne soit grillé»; ayant détaché le malheureux, on le fait «courir par la place où la jeunesse l'attend avec des tisons et des chaudronnées de cendre chaude». Chez Galinée, on «l'attachoit pieds et mains à un poteau», on le brûlait «avec un canon de fusil rouge jusqu'au milieu », «jusqu'à ce qu'il n'y eust pas en luy une seule place qui ne fust grillée», puis on le faisait «courir par la place où les Iroquois l'attendoient... plusieurs prenoient des chaudières pleines de charbons et de cendres chaudes...» ${ }^{22}$. La constance du rituel, l'existence d'une «vulgate» narrative suffiraient-elles à expliquer les ressemblances verbales? Elles nous semblent trop précises pour ne pas dénoter des réminiscences. Mais d'autres que Dollier avaient lu avec grande attention la relation de Galinée. Tout ce faisceau d'indices ne vaut pas, en effet, une preuve

20 Ibid., 342 («Un prêtre du Séminaire de Saint-Sulpice»); cf. p. 304, etc. Il lui arrive une seule fois de laisser échapper la première personne, p. 336.

21 (Éd. Margry): 118.

22 Ibid.: 177 (Récit : 135 (Galinée). 
formelle et se heurte de plus à certaines objections qu'il ne faut pas se dissimuler.

La première, tirée de l'Histoire du Montréal, peut se retourner assez aisément. Dollier, en résumant le voyage de Courcelles, déclare qu'il ne s'y attarde pas, «à cause que les RR.PP. Jésuites l'ont écrit en leur relation", et il ne fait nulle allusion à l'existence d'un autre récit. Il est amusant de constater que la Relation pour les années 1670-71 consacre, en tout et pour tout, une phrase à la «promenade» que fit le gouverneur, "accompagné des plus lestes de nos François», au pays des Iroquois. Encore cette phrase ne figure-t-elle pas dans la Relation proprement dite, mais dans la lettre d'envoi de Claude Dablon au Provincial ${ }^{23}$. Quand il écrivait son Histoire en 1672, Dollier ignorait-il encore une telle sobriété, ou ironisait-il? En tout cas, le Récit détaillé de 1671 restant inédit, il n'avait pas à en faire état, même s'il en était l'auteur.

La comparaison, poussée plus loin, fait ressortir des anomalies plus curieuses. Dans l'Histoire, Dollier signale qu'au cours du voyage, Perrot «pensa périr dans un accident de canot» 24 : or le Récit ignore cet incident. On comprend que l'historien et procureur seigneurial de Montréal ait tenu à souligner le danger couru par le gouverneur de la ville. Mais pourquoi l'aurait-il caché dans le Récit? Pourquoi la liste des officiers membres de l'expédition est-elle plus longue dans l'Histoire que dans le Récit ? ${ }^{25}$ Le désir de concentrer la lumière sur Courcelles dans un cas, sur les Montréalistes dans l'autre, rend-il compte de ces bizarreries d'un même narrateur?

De la part d'un personnage tel que Dollier, certains passages du Récit surprendraient par eux-mêmes; ainsi le préambule historique, qui souffre d'un simplisme rudimentaire. Selon l'auteur, les Français au Canada se sont d'abord contentés de s'enfermer dans des forts et de faire la traite des pelleteries, à l'abri des Iroquois. C'est, en deux lignes, méconnaitre l'action de Champlain, l'esprit de Maisonneuve, la signification de Ville-Marie. Si pressé qu'il pût être

23 Histoire du Montréal, 342. - Jesuit Relations (éd. Thwaites, réimp. 1959), 54: 38; - ou Relations des Jésuites (éd. du Jour, Montréal, 1972), VI: 2.

24 Histoire du Montréal, 342.

25 Le Récit nomme Perrot, Varennes, Loubias (183). L'Histoire ajoute Le Moine, La Vallière, Normanville (342). Ni le Récit, ni l'Histoire ne signalent que Courcelles se serait rendu aussi à la mission sulpicienne de Kenté (Quenté), comme le dit Et.-M. Faillon d'après une lettre de Marie de l'Incarnation (Histoire de la colonie française en Canada, III : 334). 
dans sa rédaction, le futur historiographe aurait-il négligemment défiguré ainsi l'image qu'il se faisait de la colonisation française? Le tableau des mœurs "sauvages" réserve une autre surprise. Après avoir noté, comme c'est l'usage, que les sauvages vivent selon leur fantaisie, sans lois, et se règlent seulement sur ce qui est estimé ou méprisé par leurs anciens, l'auteur ajoute: "C'est ce qui fait que, se réglant sur la seule loi naturelle, ils estiment le bien et haïssent le $\mathrm{mal}^{26}$." Cet optimisme philosophique n'est pas hétérodoxe en soi. Son expression sommaire cadre mal pourtant avec la prudence théologique d'un disciple de $M$. Olier qui, ailleurs, ne reconnaît guère de valeur positive à la moralité des sauvages et des Iroquois en particulier. On remarque encore que le rôle d'agents psychologiques imparti par Courcelles aux missionnaires chargés de réveiller par leurs discours la plus vive défiance entre Iroquois et Outaouais dans l'intérêt du commerce français, est défini avec un réalisme sec, dont l'expression assez cynique étonne aussi sous une telle plume: "Les Sauvages $[\ldots]$ donnèrent si bien dans le panneau... ${ }^{27}$

Bref, l'évidence interne apparaît fort sujette à caution, si l'on ajoute que le style n'apporte pas d'élément déterminant dans le débat. Il ne manque pas de vivacité, de pittoresque à l'occasion. Celui de Dollier, dans son grand ouvrage, en possède aussi, et davantage. Mais aucun trait particulièrement saillant de l'expression ne permet de trancher dans un sens ou dans l'autre.

Or un indice matériel de poids vient à la renverse de l'hypothèse Dollier. C'est l'écriture du manuscrit. ${ }^{28}$ Confrontée avec celle

26 (Récit): 175.

27 Ibid.: 191.

28 La photocopie de sept pièces autographes, dont plusieurs datées de 1672 ou 1673 , nous a été très aimablement communiquée par $M$. Bruno Harel, archiviste du Séminaire de Montréal, celle d'une lettre non datée et celle d'une attestation de 1680 par le Séminaire de Québec. L'écriture du manuscrit présente des caractéristiques affirmées (par ex., boucles inférieures très développées, très inclinées, souvent non fermées, $d$ et $v$ très contournés, etc.) qui ne se retrouvent pas chez Dollier. En signant, il écrit toujours son nom avec deux $l$, et le manuscrit écrit «Dolier» $\left(f^{\circ} 213 r^{\circ}\right)$. Une lettre de Faillon à Margry laisserait penser que la confrontation qui s'imposait, et dont fait état en termes vagues la note du manuscrit, n'a pas eu lieu: «Je n'ai aucune lettre de $M$. Dollier, répond-il (18 décembre 1852), croyant, à tort, que Margry lui en a demandé une (Lettres à P. Margry, de 1844 à 1886, présentées par L.-P. Cormier (1968), 72, 74). Le manuscrit de l'Histoire du Montréal (Bibl. Mazarine, $\mathrm{n}^{\circ} 1,963$ ) n'est pas non plus de la main de Dollier, mais offre des preuves évidentes de dictée (ainsi cette correction linéaire: «Lors que je n'en ay pas (esté moins) des tesmoins hotantiques...», 2). 
de divers autographes du sulpicien, elle se révèle au premier regard si complètement différente qu'il ne saurait être question de prendre au sérieux la note datée de 1844 qui a été citée plus haut. Le manuscrit 13.516 n'est pas «de la main» de Dollier. Il ne se présente pas, il est vrai, comme un brouillon ou un tout premier jet. Mais les corrections abondent en certaines pages. Pour considérer Dollier comme l'auteur, il faudrait donc admettre qu'il ait dicté non seulement l'état originel du récit mais aussi, sans mettre la main à la plume, les modifications et les additions qui se serrent dans les marges et parfois entre les lignes. On ne saurait nier absolument cette possibilité. On y croit très difficilement: le texte offre plutôt l'aspect d'un autographe portant les repentirs et surcharges du rédacteur.

\section{Conclusion - Les raisons d'un désintérêt}

Nous jugeons donc très imprudente l'attribution du Récit à Dollier. L'incertitude où nous demeurons, en définitive, sur l'identité de l'auteur ne sera pas tout à fait stérile si nous observons qu'elle n'est pas tout à fait fortuite. Elle découle d'un net désintérêt officiel à l'égard du voyage de Courcelles. Le Récit avait pour but de le prévenir. Il n'a pas réussi à briser une certaine conspiration du silence et il a sombré lui-même dans l'oubli.

Les Jésuites, principaux informateurs de l'opinion, ont mis une sensible mauvaise volonté à célébrer l'entreprise d'un gouverneur qu'ils n'aimaient guère, et cela d'autant plus qu'une démonstration bien plus grandiose et brillante se déroulait au même moment précisément, avec leur aide et sur le territoire de leur mission avancée. Le 14 juin, au cours d'une cérémonie qu'on a pu qualifier de "pageant ${ }^{29}$, Saint-Lusson réunissait les représentants de plusieurs tribus près de Sainte-Marie-du-Sault et leur faisait acclamer la prise de possession par le roi de France des immenses étendues de l'Ouest. C'est, bien entendu, cet événement que met en vedette la Relation de 1670-71.

Talon, hostile aux Jésuites, s'entendait plus mal encore avec Courcelles. Dès avant son retour provisoire en France en 1668, il avait projeté d'établir un poste sur le lac Ontario. Après son retour en Nouvelle-France (18 août 1670), il proposait en octobre d'en éta-

29 Louise Phelps Kellog, Early narratives of the Northwest. 1634-1699 (1917, repr. 1959), 213-220. 
blir deux et de faire naviguer sur le lac «un petit bâtiment en forme de galère ${ }^{30}$. Il était en droit de revendiquer l'initiative de l'idée et d'en juger la réalisation très inférieure à son projet. Dans les mémoires qu'il envoie au roi et à Colbert en novembre 1671, il ne souffle mot du voyage de Courcelles, ne parlant du gouverneur que pour se plaindre de lui ${ }^{31}$.

Quant à Colbert, outre qu'il accordait plus de crédit à l'intendant qu'au gouverneur, il ne devait éprouver qu'une satisfaction mitigée. Dès 1669, il avait poussé Courcelles à se montrer dans le pays des nations iroquoises, avec toutes les forces disponibles, de deux en deux ans au moins, pour les maintenir dans la crainte. En février 1671, il faisait approuver par le roi la proposition de Talon relative au navire destiné à «assurer le lac Ontario», et en écrivait ainsi à Talon et à Courcelles ${ }^{32}$. Mais dans l'intervalle Courcelles avait élevé des objections contre les directives de grand déploiement militaire en territoire iroquois, et proposé de se rendre simplement au lac Ontario avec une troupe restreinte. Le 11 mars 1671, Colbert répondait: «Puisque vous ne trouvez pas à propos de faire le voyage dans le pays des Iroquois, dont le Roi s'était remis à vous, et qu'il n'y a rien qui vous y oblige, vous pouvez vous en dispenser. Mais Sa Majesté estime qu'il n'y a rien de si important au repos de ses sujets de la Nouvelle France que de tenir toujours en crainte toutes les nations sauvages qui le pourraient troubler [...] Quant à la proposition que vous faites d'envoyer quelques compagnies d'ici pour se porter à l'entrée du lac Ontario et empêcher les courses que les Iroquois pourraient faire sur les autres nations sauvages qui sont sous la protection du Roi, Sa Majesté n'a pas estimé que cela fût nécessaire pour le bien de son service, mais elle se remet néanmoins à vous et à $\mathrm{M}$. Talon d'examiner ce qui y sera le plus convenable. ${ }^{33}$

Au reçu de cette lettre, on conçoit que Courcelles ait jugé indispensable de faire rapidement quelque chose, qui frappât davantage les esprits que le déplacement d'une petite troupe vers le lac. D'où l'idée du bâtiment plat à y faire monter par le fleuve. Ce n'était

\footnotetext{
30 Correspondance de Talon, dans le Rapport de l'Archiviste de la province de Québec (1930-31): 121, 133, 135.

${ }^{31}$ Ibid.: 155, 164, 166. Nouvelle-France. Documents historiques. Correspondance échangée entre les autorités françaises et les gouverneurs et intendants (1893), I: 202.

32 Correspondance de Talon, 146, 148.

33 Nouvelle France... I: 204.
} 
qu'un compromis original, un peu improvisé, entre les premiers désirs de Colbert et les propositions de Talon. Il ne pouvait ravir ni l'un ni l'autre. La coïncidence avec la mission de Saint-Lusson acheva d'éclipser le dernier acte important du gouvernement de Courcelles.

Cependant, si la cérémonie du 14 juin à Sainte-Marie-du-Sault flattait l'amour-propre national et la soif de gloire du roi, son bilan pratique se réduisait au néant. Au contraire, l'expérience originale et risquée, lestement menée à bien par le gouverneur et ses soixante hommes, produisit d'heureuses conséquences, indiscutables et immédiates, sur l'équilibre toujours instable de craintes, d'intérêts, de rancunes, dont dépendait la paix du pays. Cette paix, les Jésuites s'empressent de la constater et de la célébrer ${ }^{34}$, ainsi que Talon ${ }^{35}$; Frontenac cherchera à la préserver par des moyens semblables. Seul le narrateur inconnu et son récit ignoré rendaient à Courcelles la juste part qui lui revenait dans son maintien ${ }^{36}$.

34 Relation de 1670-71 (éd. Thwaites), 54: 274.

35 Mémoire du 2 novembre 1671. Correspondance, 156.

36 Marie de l'Incarnation se méprenait elle-même sur le terme du voyage, qu'elle plaçait à Quenté (Lettres, éd. Richaudeau, II: 529-530). Quant à Charlevoix, il ne le signale que brièvement à la date erronée de 1670 (Histoire et description de la Nouvelle-France, IX (éd. 1744), II : 190-191). 